Article

\title{
Reverse-Bumpy-Ball-Type-Nanoreactor-Loaded Nylon Membranes as Peroxidase-Mimic Membrane Reactors for a Colorimetric Assay for $\mathrm{H}_{2} \mathrm{O}_{2}$
}

\author{
Ying Tong, Xiangyu Jiao, Hankun Yang, Yongqiang Wen, Lei Su * and Xueji Zhang * \\ Research Center for Bioengineering and Sensing Technology, School of Chemistry and Biological Engineering, \\ University of Science and Technology Beijing, Beijing 100083, China; gailty2004@163.com (Y.T.); \\ jiaoxiangyu@126.com (X.J.); ppyhk@hotmail.com (H.Y.); wyq_wen@ustb.edu.cn (Y.W.) \\ * Correspondence: sulei@ustb.edu.cn (L.S.); zhangxueji@ustb.edu.cn (X.Z.); \\ Tel.: +86-10-8237-5840 (L.S.); +86-10-6233-3910 (X.Z.)
}

Academic Editors: Jong Seung Kim and Min Hee Lee

Received: 2 March 2016; Accepted: 29 March 2016; Published: 1 April 2016

\begin{abstract}
Herein we report for the first time fabrication of reverse bumpy ball (RBB)-type-nanoreactor-based flexible peroxidase-mimic membrane reactors (MRs). The RBB-type nanoreactors with gold nanoparticles embedded in the inner walls of carbon shells were loaded on nylon membranes through a facile filtration approach. The as-prepared flexible catalytic membrane was studied as a peroxidase-mimic MR. It was found that the obtained peroxidase-mimic MR could exhibit several advantages over natural enzymes, such as facile and good recyclability, long-term stability and easy storage. Moreover, the RBB NS-modified nylon MRs as a peroxidase mimic provide a useful colorimetric assay for $\mathrm{H}_{2} \mathrm{O}_{2}$.
\end{abstract}

Keywords: nanoreactor; flexible membrane reactor; peroxidase-mimic membrane reactor; gold nanoparticles; polydopamine

\section{Introduction}

Reverse bumpy ball (RBB)-type nanoreactors are rapidly attracting increasing interest [1,2]. The term RBB refers to a hollow porous sphere in which numerous nanoscale-sized catalysts remain supported on or partially embedded in the inner walls of the shell, in contrast to the yolk-shell structure [1]. RBB-type nanoreactors have been considered to offer additional benefits with respect to the yolk-shell countparts. For instance, the RBB-type nanoreactors can provide a greater quantity of catalytically active sites per nanoreactor [3,4]. Moreover, due to the higher contact between the catalysts and the support shell, potential synergistic effects between the catalysts and the supports may be more efficiently exploited $[1,5]$. Due to the distinctive features of the RBBs, synthesis of various RBBs and relative applications have been pursued in recent years. Nanoparticle catalysts (i.e., Au [6,7], $\mathrm{Pd}[4,8,9], \mathrm{Pt}[3,10,11], \mathrm{Mn}_{3} \mathrm{O}_{4}$ [12], and $\mathrm{Fe}_{3} \mathrm{O}_{4}$ [13]) have been attached to the inner walls of various types of porous spheres including silica $[3,4,12] \mathrm{CeO}_{2}[9,11]$ carbon [8] and polymers [7], achieving high catalytic activity.

However, the recyling process of RBB-type nanoreactors in liquid media is tedious and often laborious as a result of the required isolation by centrifugation/sedimentation or filtration $[4,14,15]$, which has hampered the recovery and reusability of the RBB-type nanoreactors in liquid media. Compared with the dispersion of catalysts in solution, thin film-type catalysts possess more favorable properties from a practical viewpoint. For instance, switching the reaction off or on through thin film catalysts is technically easier to realize, just behaving like a "dip catalyst" [16-19]. In addition, it is easier to separate thin film catalysts from reaction solutions, offering the feasibility and ease of multiple reuse [16-19]. On the other hand, recently, fabrication of flexible membrane reactors (MRs) has been pursued partly because the gained flexibility can allow the construction of catalytic reactors 
with arbitrary geometries [20-22], and great attention has been paid to preparing catalytic films on porous flexible substrates. Among common flexible materials, nylon membranes are particularly attractive $[20,21]$ due to their unique merits such as toughness, high tensile strength, elasticity, and high resistance to acids and alkalis, as exemplified by a recent study by List and co-workers, in which nylon fabric was used as a support for preparing versatile organotextile catalysts [22].

In the present study, we report fabrication of RBB-type-nanoreactor-based flexible membranes. Template carbonization method is used to synthesize the RBB-type nanoreactors. Then, the RBB-type nanoreactors are loaded on flexible nylon membranes through a facile filtration approach. The as-prepared flexible catalytic membrane as the peroxidase-mimic membrane reactors is studied. Furthermore, it is tested as a $\mathrm{H}_{2} \mathrm{O}_{2}$ sensor.

\section{Results and Discussion}

Figure 1a illustrates the synthesis process of the RBB-type nanoreactors. In Step 1, $\mathrm{SiO}_{2}$ nanospheres (NSs) were first modified with 3-aminopropyltrimethoxysilane to introduce amine groups on their surface, serving as the sacrificial core. Then, negatively charged Au NPs were deposited on the amino-functionalized $\mathrm{SiO}_{2} \mathrm{NSs}$ through the electrostatic interactions. In Step 2, the C precursor layers were coated on the surface of the $\mathrm{SiO}_{2} @ \mathrm{Au}$ NSs by the self-polymerization of dopamine, forming the $\mathrm{SiO}_{2} @ A u @$ polydopamine sandwich configuration. In Step 3, the as-obtained product was calcined in $\mathrm{N}_{2}$ atmosphere to carbonize the PDA shell $[17,23]$ and, finally, the $\mathrm{SiO}_{2}$ cores were removed by $2 \mathrm{~mol} / \mathrm{L}$ $\mathrm{NaOH}$ etching for $48 \mathrm{~h}$. The morphology of the final products was characterized by transmission electron microscope (TEM). As shown in Figure 1b, the final products exhibited the characteristic morphology of the RBB configuration: hollow NSs with NPs embedded in the inner walls of the shell.

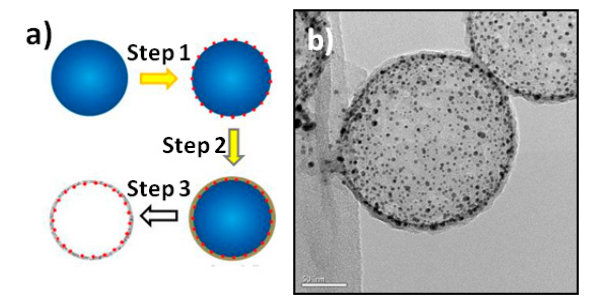

Figure 1. (a) Synthetic route to the Au NPs-embedded RBB-structured NSs. Step 1: Au loading; Step 2: PDA coating; Step 3: calcination and the core removal by alkaline etching; (b) TEM image of the RBB-structured NSs. Scale bar: $50 \mathrm{~nm}$.

Figure 2 shows a schematic illustration of the filtration-based fabrication process of the RBB-structured NSs-based catalytic flexible membrane. As illustrated, a piece of $0.20 \mu \mathrm{m}$ pore nylon membrane was inside in the filter and when the RBB NS-containing solution in the syringe was filtered through the nylon membrane, the RBB NSs were trapped within the nylon membrane, forming the RBB NS-modified nylon membrane.

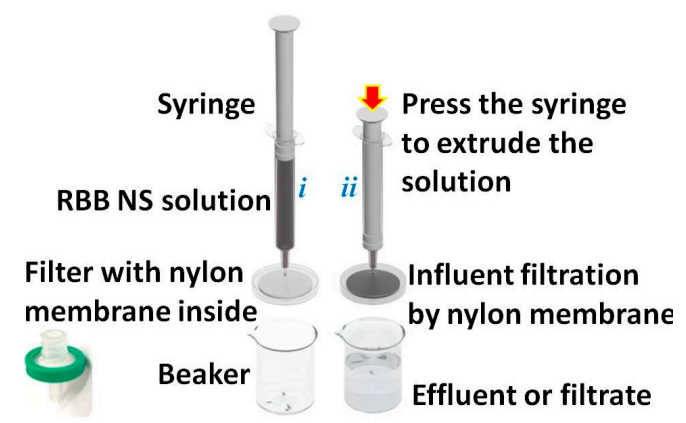

Figure 2. Schematic illustration of the filtration device (i) and the filtration process (ii). 
Success in fabrication of the RBB NS-modified nylon membrane is indicated by the visual color change of the membrane from the white before filtration to the black after filtration and by the colorless filtrate. From the plane-view scanning electron microscope (SEM) images of bare nylon membrane and the RBB NS-modified nylon membrane, shown in Figure 3a,b, respectively, it can be seen that the surface of the nylon membrane was modified with the RBB NSs. Cross-section SEM images of the RBB NS-modified nylon membrane (Figure 3c) also indicate that the RBB NSs were introduced into the nylon membrane. A low magnification SEM image of the RBB NS-modified nylon membrane indicating that large-area RBB NS-modified nylon membrane could be obtained is shown in Figure 3d. Furthermore, a high-magnification SEM image of the RBB NS-modified nylon membrane (Figure 3e) reveals that the RBB NSs should be intercepted by small pores of the nylon filter during filtration. Nylon is naturally hydrophilic and has an open pore structure, facilitating the flow of the influent through the membrane. Meanwhile, the nylon membrane is a depth filter and can retain effectively particles larger than $0.20 \mu \mathrm{m}$. As a result, the RBB NSs can be effectively immobilized within the nylon membrane. In addition, the resulting RBB NS-modified nylon membrane is not compact and still maintains the open pore structure of the nylon membrane. These structure features of the RBB NS-modified nylon membrane are favorable for mass transfer in catalytic application. Moreover, the photograph (inset of Figure 3e) shows the RBB NS-modified nylon membrane is highly flexible, with no observed change after repeated flexion.

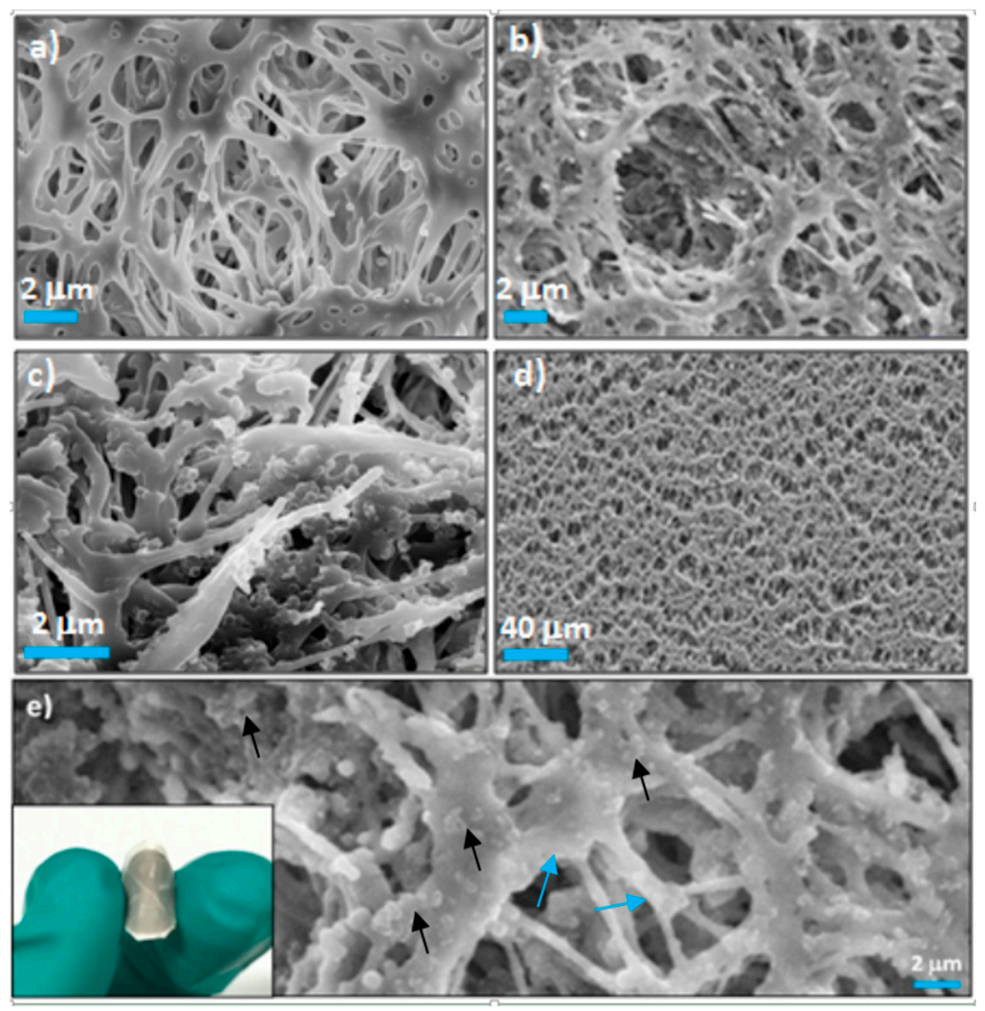

Figure 3. SEM images of the nylon (a) and the RBB-structured NS-modified nylon membranes (b-d); (e) High magnification SEM image of the RBB-structured NS-modified nylon membrane. The black arrows indicate the RBB-structured NS, and the blue arrows indicate the nylon fibre network. Inset of (e): The flexibility of the RBB-structured NS-modified nylon membrane.

To investigate the peroxidase-like activity of the RBB NS-modified nylon membrane, the catalytic oxidation of 3,3,5,5-tetramethylbenzidine (TMB), a benign and noncarcinogenic color reagent, in the presence of $\mathrm{H}_{2} \mathrm{O}_{2}$ was tested. As shown in Figure 4a, the RBB NS-modified nylon membrane could catalyze the oxidation of TMB in the presence of $\mathrm{H}_{2} \mathrm{O}_{2}$ and produce a deep blue color, with maximum 
absorbance at $650 \mathrm{~nm}$ [24]. A kinetic study showed that the RBB NS-modified nylon membrane exhibited its highest catalytic activity at approximately pH 3.5 (Figure $4 \mathrm{~b}$ ). In addition, also like natural enzymes, the peroxidase-mimic catalytic activity of the RBB NS-modified nylon membrane was dependent on temperature, showing a maximum at approximately $40{ }^{\circ} \mathrm{C}$ (Figure $4 \mathrm{c}$ ). But unlike natural enzymes, the RBB NS-based peroxidase-mimic MR could exhibit facile and good recyclability and long-term stability. The recyclability of the RBB NS-based peroxidase-mimic MR was examined by recycling the same MR. Between each cycle, the membrane was directly withdrawn from the TMB- $\mathrm{H}_{2} \mathrm{O}_{2}$ reaction solution and rinsed with deionized water. As revealed from Figure $4 \mathrm{~d}$ (bars), the MR retained almost unchanged catalytic activity towards TMB oxidation by $\mathrm{H}_{2} \mathrm{O}_{2}$ in seven successive cycles, indicating good recyclability of the RBB NS-modified nylon MR. The peroxidase-mimic activity stability test was further investigated by testing the peroxidase-mimic membrane every day. When not in use, it was stored without any other specific care at room temperature. From Figure $4 \mathrm{~d}$ (blue dotted line), it can be seen that the MR could maintain a stable catalytic activity for at least $25 \mathrm{~d}$.
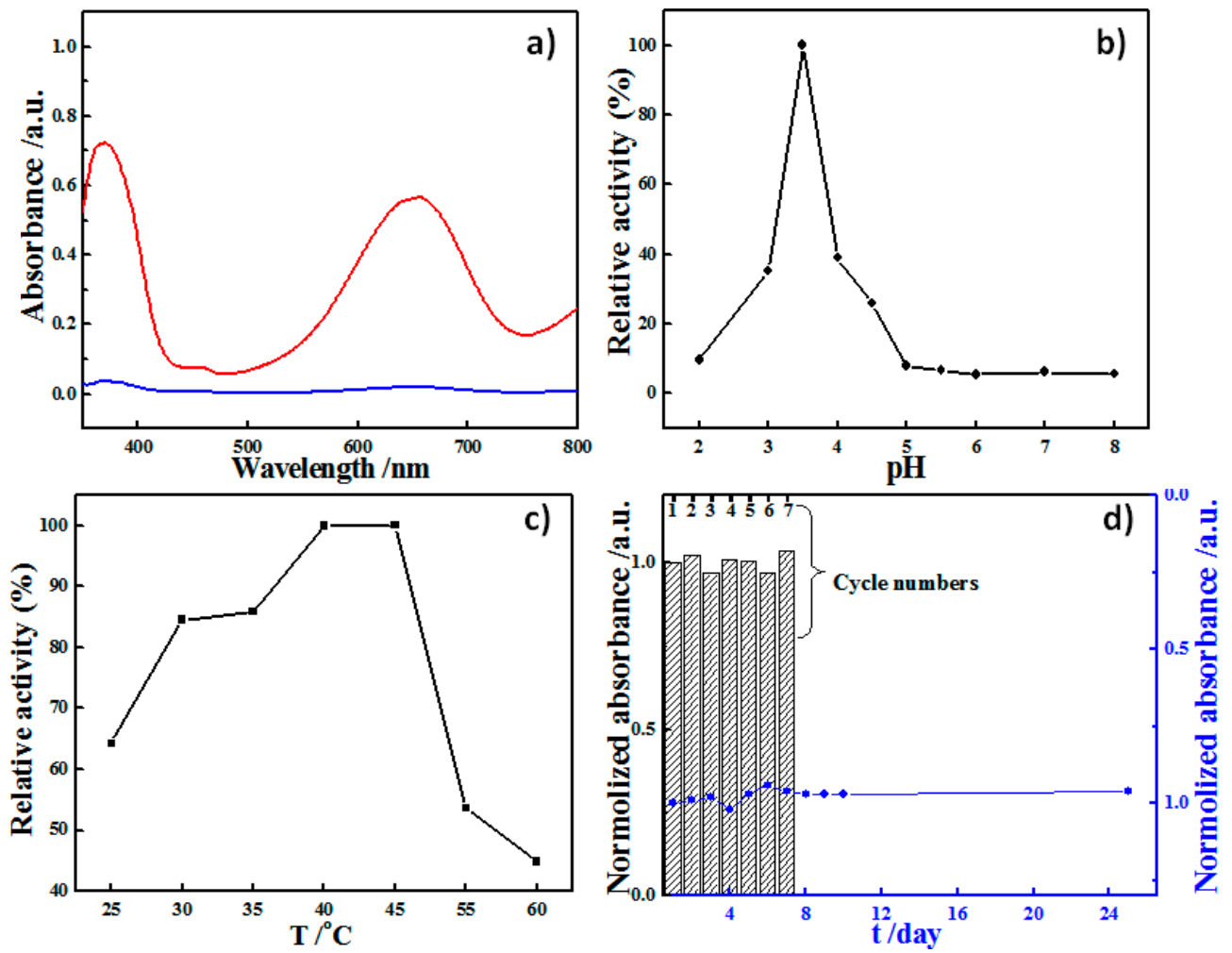

Figure 4. (a) UV-vis absorption spectra of the TMB- $\mathrm{H}_{2} \mathrm{O}_{2}$ mixture $\left(0.8 \mathrm{mmol} / \mathrm{L} \mathrm{TMB}, 50 \mathrm{mmol} / \mathrm{L} \mathrm{H}_{2} \mathrm{O}_{2}\right)$ in the absence (blue line) and presence of the RBB NS-based MR (red line) after 10 min incubation; $(\mathbf{b}, \mathbf{c})$ Plots of the peroxidase-like activity of the RBB NS-based MR against $\mathrm{pH}$ and temperature; (d) The recyclability of the RBB NS-based MR indicated by the normalized absorbance of the TMB oxidized the catalytic oxidation of $0.8 \mathrm{mmol} / \mathrm{L} \mathrm{TMB}$ by $10 \mathrm{mmol} / \mathrm{L} \mathrm{H}_{2} \mathrm{O}_{2}$ in seven successive cycles with the same MR (upper) and the long-term stability of the catalytic activity of the RBB NS-based peroxidase-like MR (bottom).

Furthermore, the catalytic activity of the RBB NS-modified nylon MR is $\mathrm{H}_{2} \mathrm{O}_{2}$ concentration dependent. As shown in Figure 5a, the absorbance of this system increased with increasing $\mathrm{H}_{2} \mathrm{O}_{2}$ concentration. Therefore, the RBB NS-modified nylon MR can be used as $\mathrm{H}_{2} \mathrm{O}_{2}$ sensor, which has potential applications in biomedical fields [25,26]. As shown in Figure 5b, under the optimal conditions (i.e., $40^{\circ} \mathrm{C}, \mathrm{pH} 3.5$ ), the absorbance at $652 \mathrm{~nm}$ was proportional to $\mathrm{H}_{2} \mathrm{O}_{2}$ concentration from 10-80 mmol/L with a detection limit of $0.8 \mathrm{mmol} / \mathrm{L}$. 

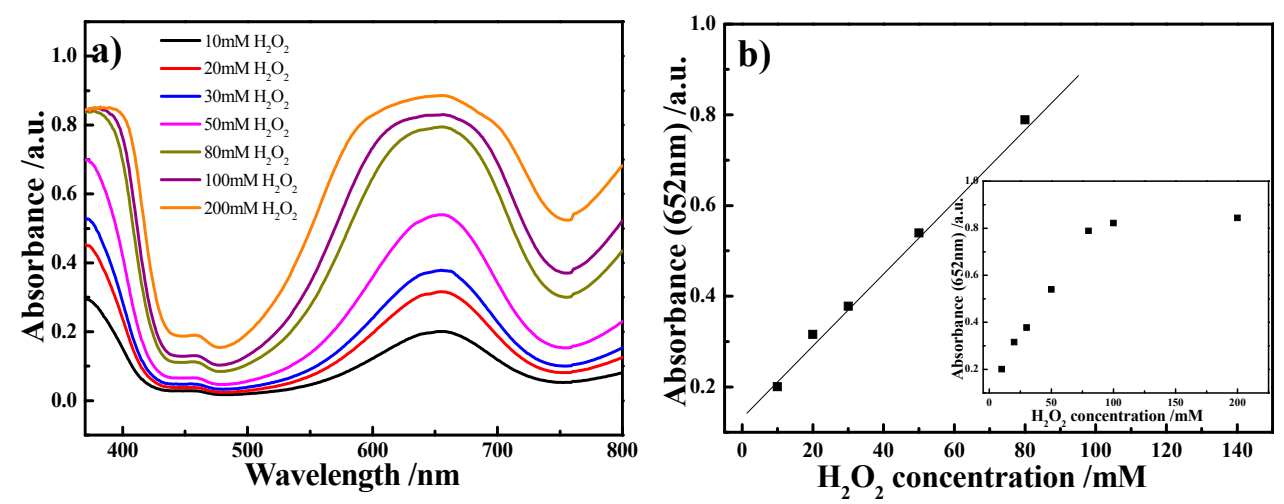

Figure 5. (a) Typical absorption spectrum of the TMB solution in the presence of $\mathrm{H}_{2} \mathrm{O}_{2}$ at various concentrations using the RBB NS-modified nylon MRs as a peroxidase mimic; (b) Linear calibration plot between the absorbance at $652 \mathrm{~nm}$ and concentration of $\mathrm{H}_{2} \mathrm{O}_{2}$. The insert shows the dependence of the absorbance at $652 \mathrm{~nm}$ on the concentration of $\mathrm{H}_{2} \mathrm{O}_{2}$ in the range $10 \mathrm{mmol} / \mathrm{L}$ to $200 \mathrm{mmol} / \mathrm{L}$.

\section{Experimental Section}

\subsection{Chemicals}

Tetraethyl orthosilicate (TEOS), (3-aminopropyl)triethoxysilane (APTS) and hydrogen tetrachloroaurate(III) hydrate were purchased from Alfa Aesar (Ward Hill, MA, USA). Dopamine chloride and 3,5,3',5'-tetramethylbenzidine (TMB) were purchased from Sigma (St Louis, MO, USA). All other chemicals of at least analytical reagent grade were obtained from Sinopharm Chemical Reagent Co., Ltd. (Beijing, China). Nylon filters $(0.20 \mu \mathrm{m})$ were purchased from Lanyi Chemical Reagent Co., Ltd. (Beijing, China). Aqueous solutions were prepared using deionized water produced by a Milli-Q water system (Millipore, Darmstadt, Germany).

\subsection{Synthesis of the RBB-Structured NSs}

$\mathrm{SiO}_{2}$ NSs (ca. $200 \mathrm{~nm}$ in diameter) were synthesized according to the Stöber method. For preparation of amino-functionalized silica NSs, $100 \mathrm{mg}$ silica NSs were dispersed in toluene, and the solution was stirred vigorously after adding $25 \mu \mathrm{L}$ APTS $(0.1 \mu \mathrm{mol} / \mathrm{L})$. Afterwards, the precipitates were collected by centrifugation, washed three times with ethanol, and then dried overnight under vacuum at $60^{\circ} \mathrm{C}$. Gold NPs (ca. $4.5 \mathrm{~nm}$ in diameter) were synthesized according to previous report [27]. To prepare the $\mathrm{SiO}_{2} @$ Au composite NSs, 20 mg amino-functionalized silica NSs were added to the above gold NP solution with stirring for $30 \mathrm{~min}$, followed by centrifugation and drying overnight under vacuum at $60{ }^{\circ} \mathrm{C}$. The obtained $\mathrm{SiO}_{2} @ \mathrm{Au}$ NSs were added into the freshly prepared $1 \mathrm{mg} / \mathrm{mL}$ dopamine tris buffer $(10 \mathrm{mmol} / \mathrm{L}, \mathrm{pH} 8.5)$ for polymerization of dopamine [28,29]. The mixture was stirred for $2 \mathrm{~h}$, followed by centrifugation, washing with deionized water and drying in vacuum at $60{ }^{\circ} \mathrm{C}$ overnight. The obtained powders were carbonized under $\mathrm{N}_{2}$ atmosphere at $500{ }^{\circ} \mathrm{C}$ for $3 \mathrm{~h}$ with a heating rate of $5^{\circ} \mathrm{C} \cdot \mathrm{min}^{-1}$. Afterwards, the powders were treated with $2 \mathrm{~mol} / \mathrm{L} \mathrm{NaOH}$ solution for $48 \mathrm{~h}$ to remove the $\mathrm{SiO}_{2}$ core, producing the final product, the RBB-structured NSs.

\subsection{Preparation of the Flexible Catalytic Membranes}

RBB NS-containing solution ( $1 \mathrm{~mL}, 0.05 \mathrm{mg} / \mathrm{mL}$ ) was transferred to a syringe and filtered with a nylon filter membrane. Then, the nylon filter membrane was washed with deionized water and ethanol, followed by drying at $60^{\circ} \mathrm{C}$ under vacuum.

\subsection{Instruments and Measurements}

The morphologies of the samples were observed using a field emission scanning electron microscopy (SEM, Supra 55, Zeiss, Oberkochen, Germany) and a field emission transmission 
electron microscopy (TEM, JEM-2100F, JEOL, Tokyo, Japan). For UV-vis absorption measurements, quartz microcuvettes with $10 \mathrm{~mm}$ path lengths and $1 \mathrm{~mm}$ window widths were used on a UV-vis spectrophotometer (UV-1800, Shimadzu, Tokyo, Japan). For measurement of TMB oxidation by $\mathrm{H}_{2} \mathrm{O}_{2}$ catalyzed by the RBB NS-based peroxidase-mimic MR, the RBB NS-modified nylon membranes were immersed in NaOAc buffer $\left(25 \mathrm{mmol} / \mathrm{L}, \mathrm{N}_{2}\right.$ saturation, $\mathrm{pH}$ 3.5) containing $\mathrm{H}_{2} \mathrm{O}_{2}$ at different concentrations and $800 \mu \mathrm{mol} / \mathrm{L} \mathrm{TMB}$. The reaction was kept at $40{ }^{\circ} \mathrm{C}$ for $10 \mathrm{~min}$. UV-vis absorption spectra were recorded to monitor the time-dependent absorbance changes at $652 \mathrm{~nm}$.

\section{Conclusions}

In conclusion, we have demonstrated the fabrication of a RBB NS-based peroxidase-mimic MR. The obtained RBB-structured NSs could be firmly captured by the nylon membrane by filtration, producing flexible membranes. The obtained catalytic membrane could be used as a peroxidase-mimic MR to catalyze the oxidation of TMB by $\mathrm{H}_{2} \mathrm{O}_{2}$ and exhibited several advantages over natural enzymes such as facile and good recyclability, long-term stability and easy storage. Moreover, the RBB NS-modified nylon MRs as a peroxidase mimic provides a colorimetric assay for $\mathrm{H}_{2} \mathrm{O}_{2}$ with a detection limit of $0.8 \mathrm{mmol} / \mathrm{L}$.

Acknowledgments: The support from the National Natural Science Foundation of China (Grant No. 21175010, 21545012 and 21275017).

Author Contributions: Lei Su and Ying Tong conceived and designed the study. Ying Tong and Xueji Zhang performed the experiments. Lei Su wrote the manuscript. Hankun Yang, Yongqiang Wen and Xueji Zhang reviewed and edited the manuscript. All authors read and approved the manuscript.

Conflicts of Interest: The authors declare no conflict of interest.

\section{References}

1. Perez-Lorenzo, M.; Vaz, B.; Salgueirino, V.; Correa-Duarte, M.A. Hollow-Shelled Nanoreactors Endowed with High Catalytic Activity. Chem. Eur. J. 2013, 19, 12196-12211. [CrossRef] [PubMed]

2. Vaz, B.; Salgueirino, V.; Perez-Lorenzo, M.; Correa-Duarte, M.A. Enhancing the Exploitation of Functional Nanomaterials through Spatial Confinement: The Case of Inorganic Submicrometer Capsules. Langmuir 2015, 31, 8745-8755. [CrossRef] [PubMed]

3. Sanles-Sobrido, M.; Perez-Lorenzo, M.; Rodriguez-Gonzalez, B.; Salgueirino, V.; Correa-Duarte, M.A. Highly Active Nanoreactors: Nanomaterial Encapsulation Based on Confined Catalysis. Angew. Chem. Int. Ed. 2012, 51, 3877-3882. [CrossRef] [PubMed]

4. Chen, Z.; Cui, Z.M.; Niu, F.; Jiang, L.; Song, W.G. Pd nanoparticles in silica hollow spheres with mesoporous walls: a nanoreactor with extremely high activity. Chem. Commun. 2010, 46, 6524-6526. [CrossRef] [PubMed]

5. Xiao, J.P.; Pan, X.L.; Guo, S.J.; Ren, P.J.; Bao, X.H. Toward Fundamentals of Confined Catalysis in Carbon Nanotubes. J. Am. Chem. Soc. 2015, 137, 477-482. [CrossRef] [PubMed]

6. Sanles-Sobrido, M.; Exner, W.; Rodriguez-Lorenzo, L.; Rodriguez-Gonzalez, B.; Correa-Duarte, M.A.; Alvarez-Puebla, R.A.; Liz-Marzan, L.M. Design of SERS-Encoded, Submicron, Hollow Particles Through Confined Growth of Encapsulated Metal Nanoparticles. J. Am. Chem. Soc. 2009, 131, 2699-2705. [CrossRef] [PubMed]

7. Liu, B.; Wang, X.M.; Zhao, Y.W.; Wang, J.C.; Yang, X.L. Polymer shell as a protective layer for the sandwiched gold nanoparticles and their recyclable catalytic property. J. Colloid Interface Sci. 2013, 395, 91-98. [CrossRef] [PubMed]

8. Zhang, Z.Y.; Xiao, F.; Xi, J.B.; Sun, T.; Xiao, S.; Wang, H.R.; Wang, S.; Liu, Y.Q. Encapsulating Pd nanoparticles in double-shelled graphene@ carbon hollow spheres for excellent chemical catalytic property. Sci. Rep. UK 2014. [CrossRef] [PubMed]

9. Du, C.H.; Guo, Y.; Guo, Y.L.; Gong, X.Q.; Lu, G.Z. Polymer-templated synthesis of hollow $\mathrm{Pd}-\mathrm{CeO}_{2}$ nanocomposite spheres and their catalytic activity and thermal stability. J. Mater. Chem. A 2015, 3, 23230-23239. [CrossRef]

10. Yin, Y.Y.; Chen, M.; Zhou, S.X.; Wu, L.M. A general and feasible method for the fabrication of functional nanoparticles in mesoporous silica hollow composite spheres. J. Mater. Chem. 2012, 22, 11245-11251. [CrossRef] 
11. Wang, X.; Liu, D.P.; Song, S.Y.; Zhang, H.J. Pt@CeO 2 Multicore@Shell Self-Assembled Nanospheres: Clean Synthesis, Structure Optimization, and Catalytic Applications. J. Am. Chem. Soc. 2013, 135, 15864-15872. [CrossRef] [PubMed]

12. Anisur, R.M.; Shin, J.; Choi, H.H.; Yeo, K.M.; Kang, E.J.; Lee, I.S. Hollow silica nanosphere having functionalized interior surface with thin manganese oxide layer: nanoreactor framework for size-selective Lewis acid catalysis. J. Mater. Chem. 2010, 20, 10615-10621. [CrossRef]

13. Zhou, D.; Yang, L.P.; Yu, L.H.; Kong, J.H.; Yao, X.Y.; Liu, W.S.; Xu, Z.C.; Lu, X.H. Fe/N/C hollow nanospheres by $\mathrm{Fe}(\mathrm{III})$-dopamine complexation-assisted one-pot doping as nonprecious-metal electrocatalysts for oxygen reduction. Nanoscale 2015, 7, 1501-1509. [CrossRef] [PubMed]

14. Liu, B.; Zhang, W.; Feng, H.L.; Yang, X.L. Rattle-type microspheres as a support of tiny gold nanoparticles for highly efficient catalysis. Chem. Commun. 2011, 47, 11727-11729. [CrossRef] [PubMed]

15. Chen, J.C.; Xue, Z.T.; Feng, S.S.; Tu, B.; Zhao, D.Y. Synthesis of mesoporous silica hollow nanospheres with multiple gold cores and catalytic activity. J. Colloid Interface Sci. 2014, 429, 62-67. [CrossRef] [PubMed]

16. Hariprasad, E.; Radhakrishnan, T.P. A Highly Efficient and Extensively Reusable "Dip Catalyst" Based on a Silver-Nanoparticle-Embedded Polymer Thin Film. Chem. Eur. J. 2010, 16, 14378-14384. [CrossRef] [PubMed]

17. Gong, W.; Su, L.; Zhang, X.J. Preparation of catalytic films of the Au nanoparticle-carbon composite tubular arrays. Chem. Commun. 2015, 51, 6333-6336. [CrossRef] [PubMed]

18. Hariprasad, E.; Radhakrishnan, T.P. Palladium Nanoparticle-Embedded Polymer Thin Film "Dip Catalyst" for Suzuki-Miyaura Reaction. ACS Catal. 2012, 2, 1179-1186. [CrossRef]

19. Faria, V.W.; Oliveira, D.G.M.; Kurz, M.H.S.; Goncalves, F.F.; Scheeren, C.W.; Rosa, G.R. Palladium nanoparticles supported in a polymeric membrane: an efficient phosphine-free "green" catalyst for Suzuki-Miyaura reactions in water. RSC Adv. 2014, 4, 13446-13452. [CrossRef]

20. Zhang, S.; Shen, X.T.; Zheng, Z.P.; Ma, Y.Y.; Qu, Y.Q. 3D graphene/nylon rope as a skeleton for noble metal nanocatalysts for highly efficient heterogeneous continuous-flow reactions. J. Mater. Chem. A 2015, 3, 10504-10511. [CrossRef]

21. Yao, J.F.; Dong, D.H.; Li, D.; He, L.; Xu, G.S.; Wang, H.T. Contra-diffusion synthesis of ZIF-8 films on a polymer substrate. Chem. Commun. 2011, 47, 2559-2561. [CrossRef] [PubMed]

22. Lee, J.W.; Mayer-Gall, T.; Opwis, K.; Song, C.E.; Gutmann, J.S.; List, B. Organotextile Catalysis. Science 2013, 341, 1225-1229. [CrossRef] [PubMed]

23. Liu, R.; Mahurin, S.M.; Li, C.; Unocic, R.R.; Idrobo, J.C.; Gao, H.J.; Pennycook, S.J.; Dai, S. Dopamine as a Carbon Source: The Controlled Synthesis of Hollow Carbon Spheres and Yolk-Structured Carbon Nanocomposites. Angew. Chem. Int. Ed. 2011, 30, 6931-6934. [CrossRef]

24. Nguyen, C.T.; Kasi, R.M. Nanocomposite hydrogels based on liquid crystalline brush-like block copolymer-Au nanorods and their application in $\mathrm{H}_{2} \mathrm{O}_{2}$ detection. Chem. Commun. 2015, 51, 12174-12177. [CrossRef] [PubMed]

25. Sánchez, S.; Pumera, M.; Cabruja, E.; Fàbregas, E. Carbon nanotube/polysulfone composite screen-printed electrochemical enzyme biosensors. Analyst 2007, 132, 142-147. [CrossRef] [PubMed]

26. Sitnikova, N.A.; Komkova, M.A.; Khomyalova, I.V.; Karyakina, E.E.; Karyakin, A.A. Transition Metal Hexacyanoferrates in Electrocatalysis of $\mathrm{H}_{2} \mathrm{O}_{2}$ Reduction: An Exclusive Property of Prussian Blue. Anal. Chem. 2014, 86, 4131-4134. [CrossRef] [PubMed]

27. Jana, N.R.; Gearheart, L.; Murphy, C.J. Wet Chemical Synthesis of High Aspect Ratio Cylindrical Gold Nanorods. J. Phys. Chem. B 2001, 105, 4065-4067. [CrossRef]

28. Bernsmann, F.; Voegel, J.C.; Ball, V. Different synthesis methods allow to tune the permeability and permselectivity of dopamine-melanin films to electrochemical probes. Electrochim. Acta 2011, 56, 3914-3919. [CrossRef]

29. Lin, Y.Q.; Hu, L.L.; Yin, L.; Guo, L. Electrochemical glucose biosensor with improved performance based on the use of glucose oxidase and Prussian Blue incorporated into a thin film of self-polymerized dopamine. Sens. Actuators B Chem. 2015, 210, 513-518. [CrossRef]

(c) 2016 by the authors; licensee MDPI, Basel, Switzerland. This article is an open access article distributed under the terms and conditions of the Creative Commons by Attribution (CC-BY) license (http:/ / creativecommons.org/licenses/by/4.0/). 